\title{
Corrigendum
}

\section{Generalized Slavnov-Taylor, BRST and covariance identities from the geometry of the gauge surface}

\author{
P.D. Jarvis ${ }^{1}$ and G. Thompson ${ }^{2}$ \\ ${ }^{1}$ Department of Physics, University of Tasmania, Box 252C, GPO, Hobart, Tasmania, 7001, Australia \\ ${ }^{2}$ International Centre for Theoretical Physics, I-34100 Trieste, Italy, \\ Z. Phys. C - Particles and Fields 26, 315-21 (1984) \\ Received 6 May 1987
}

\begin{abstract}
We establish the equivalence between the extended BRST invariances, and the conventional Slavnov-Taylor transformations together with a new "dual" analogue. However, the latter (a non-local gauge transformation, generating an $A$-dependent translation of the gauge-fixing surface) is not an invariance of the Faddeev-Popov determinant, contrary to the published claim.
\end{abstract}

In our previous paper it was demonstrated that properties such as the Slavnov-Taylor and covariance identities, of central importance in many aspects of quantized gauge theories, could be systematically viewed in terms of translations, rotations and dilations in the space of components of the gauge-fixing constraint, e.g. $L=\partial \cdot \mathrm{A}$ in covariant gauges.

The Slavnov-Taylor transformations

$A_{\mu}^{\prime}=A_{\mu}+D_{\mu}(\partial \cdot D)^{-1} A$,

in the augmented generating functional

$$
\begin{aligned}
Z\left[J_{\mu}, J, \bar{J}\right]= & N \int[d A] \Delta(A) \exp i \int d x\left(L-L^{2} / 2 \alpha\right. \\
& \left.+\bar{J}(\partial \cdot D)^{-1} J-J_{\mu} A^{\mu}\right),
\end{aligned}
$$

gave rise to a local identity reflecting the BRST-invariance of the equivalent gauge-fixing plus ghost functional

$$
\begin{aligned}
Z\left[J_{\mu}, J, \bar{J}\right]= & N \int[d A][d \omega][d \bar{\omega}] \\
& \cdot \exp i \int d x\left(L-L^{2} / 2 \alpha+\bar{\omega}(\partial \cdot D) \omega\right. \\
& \left.-\bar{J}_{\mu} A^{\mu}-\bar{J} \omega-J \bar{\omega}\right)
\end{aligned}
$$

a new "dual" Slavnov-Taylor transformation

$A_{\mu}^{\prime}=A_{\mu}+D_{\mu}(D \cdot \partial)^{-1} A$

was identified as being responsible for the "dual" BRST invariance of $Z\left[J_{\mu}, J, \bar{J}\right]$ (wherein the roles of ghosts and antighosts are essentially interchanged). However, contrary to the published claim, (4) is not an invariance of the Faddeev-Popov determinant. Instead the variation provides an essential extra term required for establishing that the resulting gauge identity is just the dual BRST identity. The analysis presented below replaces the appendix of our previous paper, together with that part of Sect. 3 (ii) of that paper pertaining to the "dual" Slavnov-Taylor identity.

The Faddeev-Popov determinant reflects gauge transformations near the gauge-fixing surface:

$\Delta(A)=\operatorname{det} F, \quad F^{a b}=\delta L^{a} / \delta g^{b}$

where $\left(g^{a}\right)$ are infinitesimal group parameters. For covariant gauges we have $F=(\partial \cdot D)$. Corresponding to (4) we have

$L^{\prime}=L+F\left(F^{T}\right)^{-1} \Lambda$

and accordingly we find

$$
\begin{aligned}
\Delta^{\prime}= & \Delta \exp \left(\Lambda \mathrm{F}^{-1}\right)^{d}\left\{\left(F^{-1}\right)^{a c} \delta F^{d c} / \delta g^{b}\right. \\
& \left.-\delta F^{d c} / \delta \mathrm{g}^{b}\left(F^{-1}\right)^{c a}\right\} \cdot \delta^{a b} .
\end{aligned}
$$

Introducing space-time indices $s, t, u, \ldots, z$ corresponding to the internal labels $a, b, c, \ldots, h$ we have 
explicitly

$$
\begin{aligned}
\Delta^{\prime}= & \Delta \exp A_{w}^{e}\left(\partial \cdot D^{-1}\right)_{w v}^{e d}\left(\partial \cdot D^{-1}\right)_{t u}^{b c} \\
& \cdot\left\{f^{c h d} \partial_{u} \cdot\left(D_{u t}^{h b} \delta_{u v}\right)-f^{d h b} \partial_{v} \cdot\left(D_{v u}^{h c} \delta_{v t}\right)\right\}
\end{aligned}
$$

with summation (or integration) on repeated indices. Here we write

$\left(D_{\mu}\right)_{s t}^{a b}=\partial_{\mu}^{s} \delta_{s t} \delta^{a b}+f^{a h b} A_{\mu}^{h}(s) \delta_{s t} \equiv\left(D_{\mu}\right)_{s}^{a b} \delta_{s t}$.

In the expansion of the first term in the expression in braces in (5) we have

$\partial_{u} \cdot\left(D_{u t}^{h b} \delta_{u v}\right) \equiv(\partial \cdot D)_{u t}^{h b}-D_{u t}^{h b} \cdot \partial_{v} \delta_{u v}$.

Inserting appropriate $\delta$ functions to replace $(\partial \cdot D)\left(\partial \cdot D^{-1}\right)$ in the first term of $(5)$ gives

$\left(\Lambda \partial \cdot D^{-1}\right)_{v}^{d} \delta^{h c} \delta_{u v} f^{c h d} \delta_{u v} \equiv 0$

by virtue of the antisymmetry of the structure constants. After integrating by parts in the second terms of (5) and (7), performing the indicated integrations over $t$ and $u$, respectively, the exponent is

$$
\begin{aligned}
& \partial_{v}\left(\Lambda \partial \cdot D^{-1}\right)_{v}^{d}\left\{f^{c h d} D_{v t}^{h b}\left(\partial \cdot D^{-1}\right)_{t v}^{b c}\right. \\
& \left.+f^{d h b} D_{v t}^{h c}\left(\partial \cdot D^{-1}\right)_{v t}^{b c}\right\}
\end{aligned}
$$

The two terms in braces simply correspond to the application of $D_{v}$ to $\left(\partial \cdot D^{-1}\right)_{v v}^{b c}$. Integration by parts gives

$$
\begin{aligned}
& f^{b c d}\left(\partial \cdot D^{-1}\right)_{v v}^{b c}(D \cdot \partial)_{v t}^{d h}\left(\Lambda \partial \cdot D^{-1}\right)_{t}^{h} \\
& \equiv f^{b c d}\left(\partial \cdot D^{-1}\right)_{v v}^{b c}\left(\Lambda \partial \cdot D^{-1}\right)_{t}^{h}(\partial \cdot D)_{t v}^{h d}
\end{aligned}
$$

yielding the final form for the variation,

$\Delta^{\prime}(A)=\Delta(A) \exp f^{a b c}\left(\partial \cdot D^{-1}\right)_{x x}^{a b} \Lambda_{x}^{c}$

Turning now to the gauge identity for $Z\left[J_{\mu}, J\right.$, $\bar{J}]$ based on the "dual" Slavnov-Taylor transformation (4) we firstly note that in (2),

$$
\begin{aligned}
\delta\left(\bar{J}(\partial \cdot D)^{-1} J\right) & =-\delta\left(J(D \cdot \partial)^{-1} \bar{J}\right) \\
& =+\left(J D \cdot \partial^{-1}\right) \delta(D \cdot \partial)\left(D \cdot \partial^{-1} \bar{J}\right)
\end{aligned}
$$

$$
\begin{aligned}
= & \left(J D \cdot \partial^{-1}\right)_{s}^{a} \cdot f^{a e b} D_{s u}^{e c} \cdot \partial_{s} \delta_{s t} \\
& \cdot\left(D \cdot \partial^{-1} A\right)_{u}^{c} \cdot\left(D \cdot \partial^{-1} \bar{J}\right)_{t}^{b}
\end{aligned}
$$

Choosing $\Lambda=-\varepsilon \bar{J}$ and performing the indicated differential operations,

$\delta\left(\bar{J}(\partial \cdot D)^{-1} J\right)=\varepsilon\left(\partial \cdot D^{-1} J\right) \cdot D\left(D \cdot \partial^{-1} \bar{J}\right) \times \partial\left(D \cdot \partial^{-1} \bar{J}\right)$

where all quantities are local and the scalar triple product has been introduced. After integration by parts we find *

$$
\begin{aligned}
\delta\left(\bar{J}(\partial \cdot D)^{-1} J\right)= & \varepsilon\left(-\bar{J} \cdot\left(\partial \cdot D^{-1} J\right) \times\left(D \cdot \partial^{-1} \bar{J}\right)\right. \\
& +\frac{1}{2} J \cdot\left(D \cdot \partial^{-1} \bar{J}\right) \times\left(D \cdot \partial^{-1} \bar{J}\right) .
\end{aligned}
$$

For the remaining terms in (2) under the change of variables (4) we not $L \cdot(\partial \cdot D)=L \cdot(D \cdot \partial)$ so

$\delta L^{2}=2 \Lambda L$

so including (8) the gauge identity can be expressed as

$$
\begin{aligned}
N \int d[A] & \int\left\{\bar{J} L(A) / \alpha+J^{\mu} D_{\mu}\left(D \cdot \partial^{-1} \bar{J}\right)\right. \\
& +\frac{1}{2} J \cdot\left(D \cdot \partial^{-1} \bar{J} \times\left(D \cdot \partial^{-1} \bar{J}\right)\right. \\
& -\bar{J} \cdot\left(\partial \cdot D^{-1} J \times D \cdot \partial^{-1} \bar{J}\right) \\
& \left.+i f^{a b c}\left(\partial \cdot D^{-1}\right)_{x x}^{a b} \bar{J}_{x}^{c}\right\} \cdot \Delta e^{i S}
\end{aligned}
$$

where a factor of $i \varepsilon$ has been dropped. But (10) is in precisely the right form to be written entirely in terms of $\Sigma$ (source) (source derivative) acting on $Z$ : in particular the last term arises from the cross term in the application of $\bar{J} \cdot i \delta / \delta \bar{J} \times i \delta / \delta J$ to the Gaussian $\exp i \bar{J}(\partial \cdot D)^{-1} J$. As claimed, this is the signal for the dual BRST invariance of the gauge-fixing plus ghost action (3).

$$
\begin{aligned}
& \delta A_{\mu}=\varepsilon D_{\mu} \bar{\omega}, \\
& \delta \bar{\omega}=-\frac{1}{2} \varepsilon \bar{\omega} \times \bar{\omega}, \\
& \delta \omega=-\varepsilon(\omega \times \bar{\omega}+L(A) / \alpha) .
\end{aligned}
$$

$\overline{\star \chi} \chi \cdot \bar{\chi} \times \partial \bar{\chi}=-\chi \cdot \bar{\chi} \times D \cdot \partial \bar{\chi}-D \chi \cdot \bar{\chi} \times \partial \bar{\chi}=$

$-\chi \cdot \bar{\chi} \times D \cdot \partial \bar{\chi}+\frac{1}{2} \partial \cdot D \chi \cdot \bar{\chi} \times \bar{\chi}$ where in the second form we use $\bar{\chi} \times \partial \bar{\chi}=\frac{1}{2} \partial(\bar{\chi} \times \bar{\chi})$ 